\title{
Research on Compensation Technology of Automobile Panel Die Surface Based On Reverse Engineering
}

\author{
Chen Zhang', Peng Du ${ }^{2}$ \\ ${ }^{1}$ Tianjin Engineering Institute of Mold Digital Manufacturing Technology, Tianjin University of Technology and Education No.1310, Dagu \\ South Road, Hexi District, Tianjin 300222, China \\ ${ }^{2}$ Tianjin Stanley Electric Science and Technology Co., Ltd, No. 99 Sixth Street, economic and Technological Development Zone, Tianjin, \\ China
}

\begin{abstract}
Aiming at the problem of the automobile panel die surface compensation technology which are difficult to guarantee quickly and occupies most of the processing cycle, the method of data analysis based on reverse engineering is put forward. The main steps: first point cloud acquisition by non-contact measurement; secondly point cloud pretreatment; and data model comparison in Geomagic qualify; Section deviation value obtained by $3 D$ and $2 D$ compared. Fitter to compensate the deviation values and rules are obtained. Die face compensation equation are established to achieve rapid acquisition mode of the compensation amount. This method is used to determine the compensation amount of the car door. The results show that the method can quickly and accurately determine the amount of compensation which provides theoretical basis for the new mold in the future development.
\end{abstract}

Keywords: Reverse engineering, Automobile panel, Die surface compensation, Mold closing rate

\section{Introduction}

With the rapid development of the automobile manufacturing industry, the replacement speed of the vehicle is accelerated gradually. The quality requirements of the mold surface about automobile covering parts are also getting higher and higher. In the light of the automobile cover parts die face fitting rate stability requirements, the traditional way is the coloring method which applied Hongdan powder or blue oil in both sides of the products. The level of mold closing rate is determined by the left in the product with color patches after closing the mold. But this method need repeated trials of the bench, and extend the production cycle. Each mold repair deviation is different, and it don't have universal ${ }^{[1]}$.

In view of the above situation, domestic and foreign research is mainly CAE simulation or coloring method to confirm the mold closing rate. But all kinds of methods need to trial repeatedly, and they cannot quickly determine the die face compensation amount. X Xin, P Lu, K Jiang ${ }^{[2]}$ proposed a method based on one step forming and geometric correction. The model is obtained by reverse engineering. The springback is simulated and calculated. X Liu, GE Xijun, $\mathrm{P} \mathrm{Liu}^{[3]}$ are adopted to compensate the springback by software ETA/Dynaform5.5, and the final dimension and angle of springback are obtained by section method. The researchers have also used the simulation method, but the simulation results can not reflect the actual rebound. Gui Li, Yuqi Liu, Ting $\mathrm{Du}^{[4]}$ proposed a geometric rebound compensation algorithm based on displacement adjustment. A new definition of the user defined characteristics. But due to the complexity of the algorithm, it is difficult to achieve a wide range of promotion. Therefore, it is necessary to develop a rapid and accurate method of die surface compensation for automobile panels. In this paper, based on the reverse engineering, laser scanning is carried out on the door panel die after compensation of automobile panel. After the point cloud processing technology, the data is compared with the original model to determine the variation of the deviation and the model surface compensation equation.

\section{Reverse Engineering Technology}

Reverse engineering technology including point cloud acquisition, point cloud pre-processing, surface reconstruction, surface fairing technology etc. The point cloud acquisition method: contact measurement and non-contact measurement.

Laser scanning technology is a non - contact measurement. The equipment has the laser scanner, the flexible arm and so on. The advantage are no damage to the work piece, clamping and convenient operation, suitable for measuring complex surface, quickly and accurately obtaining the parts of point cloud. The automobile door plate as an example, hand-held laser scanner point cloud acquisition tool is used. Obtained point cloud data as shown in figure 1.

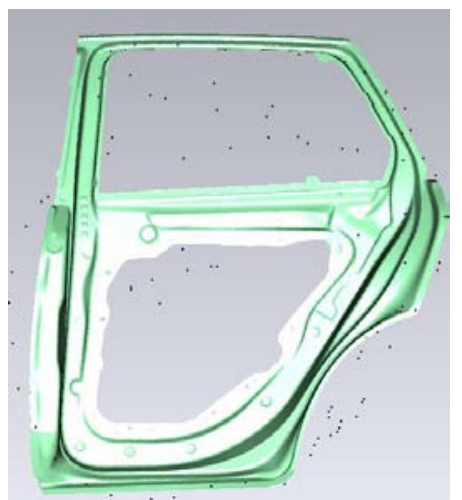

Figure 1: Original point cloud data

A large number of point cloud is obtained by non-contact measurement which reduce the computer calculation speed. Point cloud has error noise points. And point cloud smoothing 


\section{International Journal of Science and Research (IJSR) \\ ISSN (Online): 2319-7064 \\ Index Copernicus Value (2013): 6.14 | Impact Factor (2014): 5.611}

degree did not reach the surface requires. Therefore, point cloud requirements are achieved by pretreatment. Data preprocessing included the removal of noise, smoothing, data sampling and so on ${ }^{[5]}$. Figure 2 shows the data after the point cloud pretreatment.

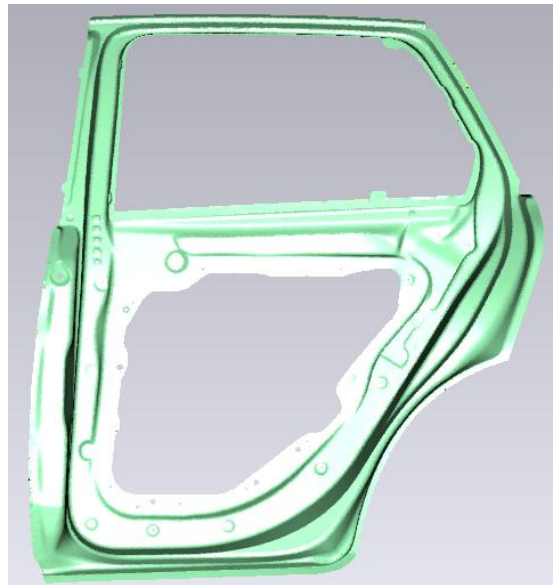

Figure 2: Post processing point cloud data

\section{Data Model Comparison}

Because the coordinates of the point cloud depends on the coordinate of the measuring equipment. And the original model is located in the design coordinate. It needs to unify the point cloud and the numerical model to the same coordinates through the coordinate transformation, and then carry on the numerical simulation ${ }^{[6]}$. Alignment technology is divided into coarse and fine alignment.

Coarse alignment use three point method which is collected three or more than three common point coordinates respectively. According to the relationship between the point coordinates, the rotation transformation matrix of two sets of points is obtained by using the four element method or the least square method. These methods are simple to operate, easy to observe, and to achieve the purpose of coarse alignment. Precision alignment method is the ICP algorithm which overlaps iteration between cloud and module. According to the best fitting principle, the two rotation transformation matrix is established. So that the point cloud and module can get a better alignment effect. ICP algorithm can make the point cloud and module to achieve the best fitting. And it can reduce the distance between the point cloud and module of the error.

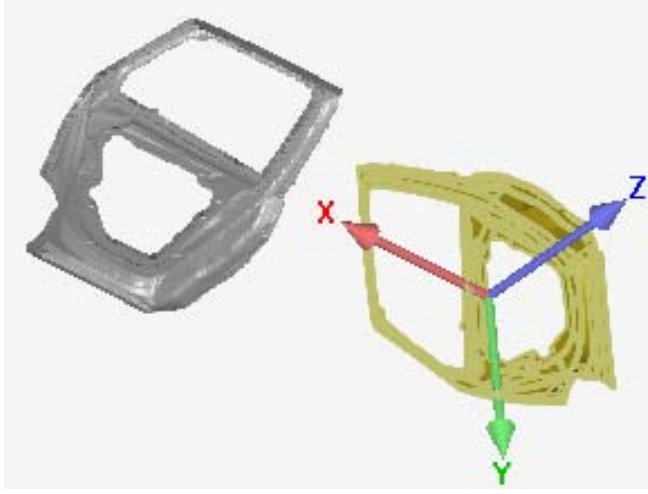

Figure 3: Original point cloud data

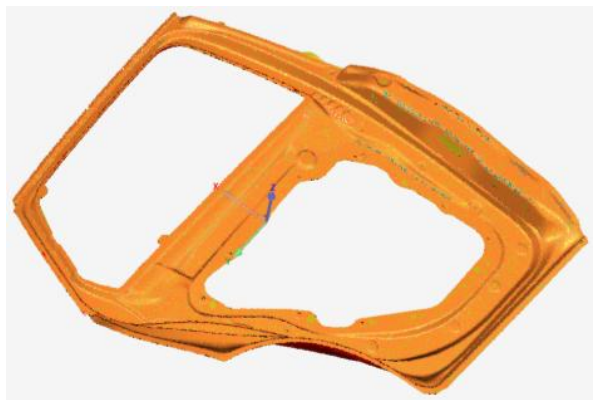

Figure 4: Post processing point cloud data

The data model comparison of automobile panel die is that the point cloud data of automobile panel die is compared with the original CAD model. At the first, 3D comparison is used. 3D comparison results are used in the interception of $2 \mathrm{D}$ profile to complete 2D comparison. And then the deviation and die surface compensation is found out.

\subsection{D Comparison}

The point cloud and the module are introduced into the Geomagic Qualify software. The deviation of the cloud and the original model is calculated by using the original data model as the reference object. 3D comparison results are display by the chromatogram. As shown in Figure 5, the blue area indicates a large deviation results.

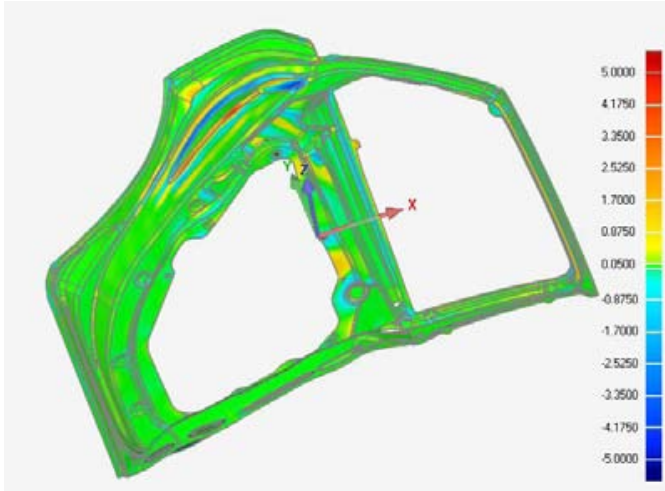

Figure 5: 3D comparison results

\subsection{D Comparison}

A section is created in the Geomagic Qualify software. The section distance of the point cloud and module is analyzed, and the cross section deviation diagram is obtained. Figure 6 is the $2 \mathrm{D}$ section deviation comparison results which is in the Figure 5 blue region $X=-143.00 \mathrm{~mm}$. Table 1 is the corresponding deviation table. 


\section{International Journal of Science and Research (IJSR) \\ ISSN (Online): 2319-7064}

Index Copernicus Value (2013): 6.14 | Impact Factor (2014): 5.611

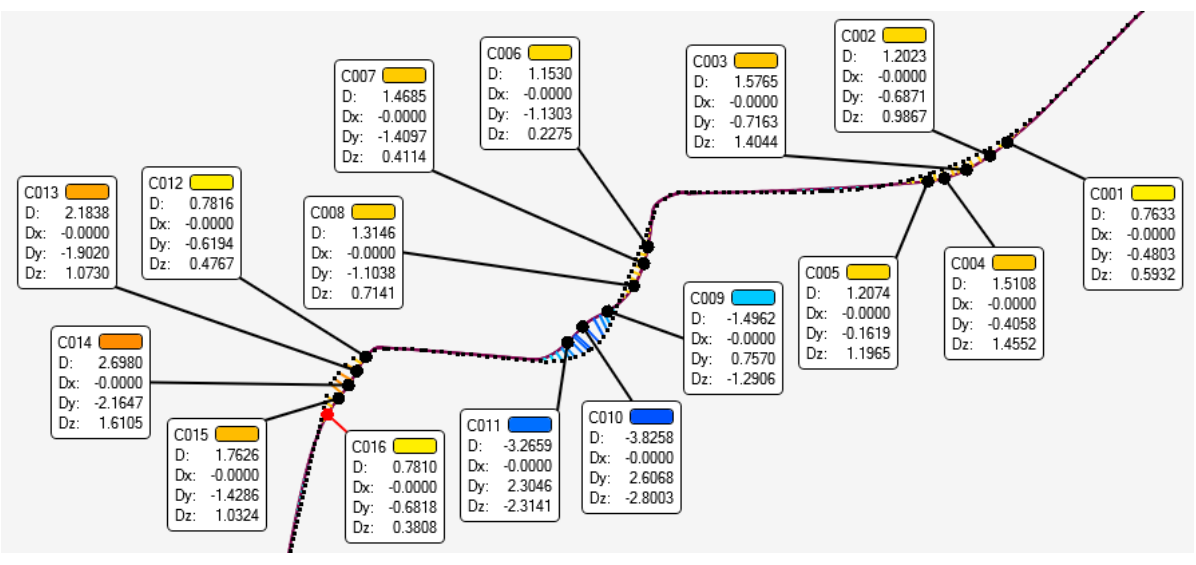

Figure 6: 2D comparison results

Table 1: 2D section deviation table of $\mathrm{YZ}=-143 \mathrm{~mm}$

\begin{tabular}{|c|c|c|c|c|c|c|}
\hline Name & reference $X$ & reference $Y$ & reference $Z$ & Measured X & Measured Y & Measured Z \\
\hline C001 & -143.0000 & 449.4050 & 109.5136 & -143.0000 & 448.9248 & 110.1069 \\
\hline C002 & -143.0000 & 446.4540 & 107.2723 & -143.0000 & 445.7670 & 108.2589 \\
\hline C003 & -143.0000 & 442.8346 & 105.0869 & -143.0000 & 442.1183 & 106.4913 \\
\hline C004 & -143.0000 & 438.8820 & 103.5342 & -143.0000 & 438.4761 & 104.9894 \\
\hline C005 & -143.0000 & 436.3976 & 103.0191 & -143.0000 & 436.2358 & 104.2156 \\
\hline. &. &. &. &. &. &. \\
\hline. &. &. &. &. & &. \\
\hline. &. &. & &. &. \\
\hline
\end{tabular}

\section{Research Method of Die Surface Compensation}

The compensation amount is a vector with a direction in the die surface modification. So it need to determine the compensation change relation of different shape and curved surface. The die surface compensation equations of different shapes and surfaces are established respectively. The shape of the mold surface of the automobile panel is complex. The acquisitions of 5 points are obtained by 2D comparison which is the fillet compensation deviation. The compensation equation of the position is fitted. And the compensation law of the original mold is obtained. According to table 1 , the collected data before and after compensation is displayed. Fillet C001-C005 compensation at the $\mathrm{Y}$ and $\mathrm{Z}$ direction of the fitting curve as shown in Figure 7,8. According to the position of the point on the curve, figure 7 equation synthetic quadratic equation can make the point falls on the surface. Figure 8 equation synthetic cubic equations can make the point fall on the surface.

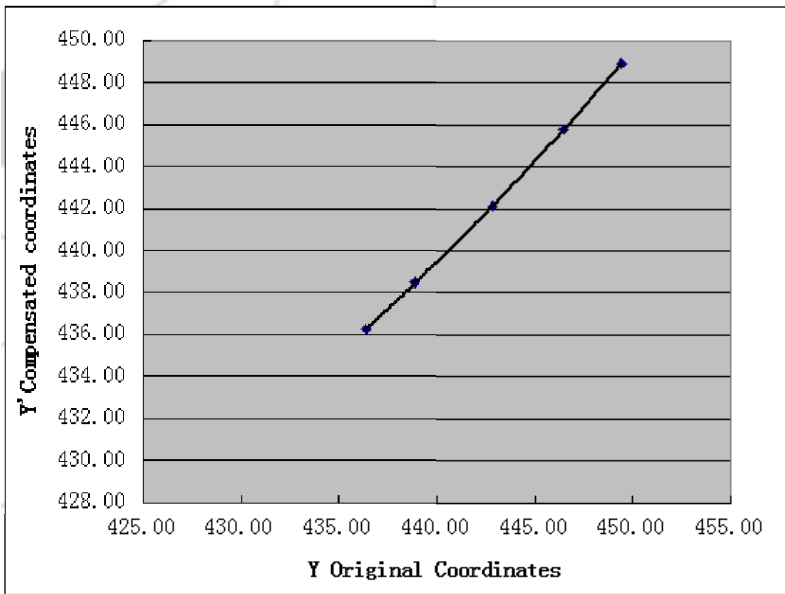

Figure 7: Y to compensate change law 
International Journal of Science and Research (IJSR)

ISSN (Online): 2319-7064

Index Copernicus Value (2013): 6.14 | Impact Factor (2014): 5.611

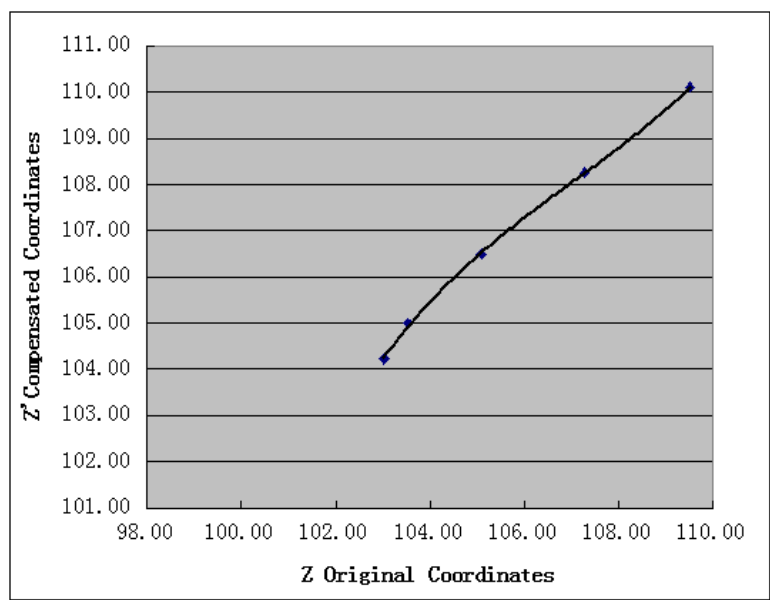

Figure 8: Z to compensate change law

Equations derived from the variation of $\mathrm{Y}$ coordinates:

Fillet A: $Y^{\prime}=0.0091 Y^{2}-7.0813 Y+1794.8$

Equations derived from the variation of $\mathrm{Z}$ coordinates:

Fillet A: $Z^{\prime}=0.0131 Z^{3}-4.215 Y^{2}+451.79 Y-16060$

By fitting the compensation equation of die surface, the car cover parts die surface compensation amount is found out. By this method also can establish different models of different sheet metal parts stamping die surface compensation amount which created a theoretical basis for follow-up study of new models and new mold development.

\section{Conclusion}

The determination method of the compensation amount of automobile panel die surface and the classification scheme based on data analysis are determined. Firstly, the car door panel mold surface 2D comparison results is collected. The model surface compensation equation is obtained by interpolation. The compensation equation of different positions in different coordinates of different sections is obtained. This method is easy to use and reference the compensation of similar mold in new mold production. The compensation amount of die surface is determined, which greatly shortens the processing cycle of the die.

\section{Acknowledgement}

This work is supported by Tianjin Universities" Training Plan of Subject leading Personnel"(RC14-02), Major Project of Tianjin Science and Technology Support (14ZCZDGX00002) and Tianjin Science and Technology Support Project(13ZCZDGX01108).

\section{References}

[1] Y D Liu, H S Xie, Y Wu, "Modulus ratio in the complex mold surface detection", Manufacturing technology and machine tool, (8)pp.131-135, 2015.

[2] X Xin, P Lu, K Jiang, eta. "Reversing factor springback compensation method based on reverse engineering technology", Computer Integrated Manufacturing Systems, CIMS, 21(6) pp. 1436-1441, 2015.

[3] X Liu, GE Xijun, P Liu, eta, “Stamping springback and springback compensation of the automobile central channel", Materials Science \& Technology, 23(4)pp. 36-41, 2015.

[4] Gui Li, Yuqi Liu, Ting Du, eta, “Algorithm research and system development on geometrical springback compensation system for advanced high-strength steel parts", The International Journal of Advanced Manufacturing Technology,70(1-4)pp. 413-427, 2014.

[5] Luo Dabing, Gao Ming, Wang Peijun, "Digital measurement in reverse engineering Volume and point cloud data processing", Mechanical Design and Manufacture, (9)PP. 56-58, 2005.

[6] L X Fan, "Research on multi view point cloud registration and surface registration in CATIA environment", Shenyang: Shenyang University of Aeronautics and Astronautics, 2014.

\section{Author Profile}

Chen Zhang is reading the M.S. degrees in Mechanical Engineering from Tianjin University of Technology and Education. 